\title{
Investigação em malária em Portugal: Mapeando modos diversos de fazer ciência
}

\begin{abstract}
Ana Ferreira ${ }^{1}$
\section{Resumo}

A ciência realizada em Portugal tem contribuído para um aprofundamento do conhecimento da malária, uma patologia responsável por 584 mil mortes em 2013. Partindo do mapeamento exploratório das publicações indexadas na Web of Science (1900-2014) acompanhar-se-ão as transformações das práticas científicas num locus de legitimação internacional. Verificou-se um crescimento acentuado destas publicações, principalmente após 1995; um favorecimento das colaborações internacionais e um alargamento sucessivo dos contextos organizacionais, significativamente superior nas publicações internacionais. Assim, o crescimento da investigação em malária é dominado por um modelo de internacionalização responsável por um imbricamento crescente da academia como os domínios político, económico e social.
\end{abstract}

Palavras-Chave: Ciência; Malária; Internacionalização; Heterogeneidade organizacional

\section{Malaria research in Portugal: Mapping diverse modes of doing science}

\begin{abstract}
Scientific research in Portugal has contributed to deepen the knowledge on malaria, a pathology responsible for 584 thousand deaths in 2013. The exploratory mapping of Portugal-affiliated papers indexed in Web of Science between 1900 and 2014 allowed characterizing scientific practices' transformations in a locus of international legitimization. This analysis revealed the growth of malaria publications, mainly after 1995; the predominance of international coauthorships; and an increasing organizational heterogeneity, significantly more pronounced in international publications. Thus, the growth of malaria research was fostered by an internationalization model responsible for an increasing intertwining of academia in political, economic, and social domains.
\end{abstract}

Keywords: Science; Malaria; Internationalization; Organizational heterogeneity

1 Centro Interdisciplinar de Ciências Sociais CICS.NOVA - Faculdade de Ciências Sociais e Humanas Universidade Nova de Lisboa (CICS.NOVA.FCSH/UNL), Avenida de Berna, 26 C, 1069-061, Lisboa, Portugal 


\section{Introdução}

Em 2013, a malária foi responsável pela morte de 584 mil pessoas. Nesse mesmo ano, $45 \%$ da população mundial encontrava-se sob risco de desenvolver esta patologia - A malária é assim um enorme risco para saúde pública, mais acentuado nas geografias onde a doença é endémica. Este não é actualmente o caso de Portugal, onde o paludismo foi, em 1973, declarado erradicado pela organização mundial de saúde . Os casos que actualmente ocorrem em território nacional são maioritariamente importados de regiões endémicas, particularmente dos países de língua oficial Portuguesa .

Os impactos globais da malária e a proximidade histórica e territorial a esta patologia, tanto em Portugal como nos países de língua oficial Portuguesa (países com quem os cientistas Portugueses mantêm relevantes colaborações científicas nas mais diversas áreas do conhecimento ), não serão despiciendos da concretização empírica de investigação científica em malária em Portugal desde há longa data. Instituições como a Escola de Medicina Tropical de Lisboa, fundada em 1902, e o seu actual "sucessor", o Instituto de Higiene e Medicina Tropical, o Instituto de Malariologia de Águas de Moura (1934), entre outras, apresentaram contributos significativos para uma compreensão mais aprofundada do paludismo. Se estas instituições foram centrais para a caracterização dos mecanismos patofisiológicos que subjazem ao desenvolvimento da malária, o reconhecimento social desta investigação científica advém também da projecção social de figuras centrais da medicina e ciência em Portugal como Ricardo Jorge, Francisco Cambournac ou, actualmente, Maria Mota, entre múltiplos outros. No entanto, se a relevância científica e social dos contributos dos cientistas Portugueses são incontornáveis no domínio das ciências da saúde, esta situação não parece ter paralelo em estudos sociais da malária, que, salvo raras excepções de trabalhos recentes com um enfoque central diverso do da presente investigação, permanece, em larga medida, por realizar.

Este será pois o principal contributo do corrente trabalho. Um primeiro mapeamento e caracterização exploratória da investigação científica em malária realizada parcial ou totalmente em instituições Portuguesas desde o início do século XX. Este estudo inevitavelmente acompanhará as transformações da produção de conhecimento científico, mudanças que se têm vindo a revelar, mais acentuadamente, desde os anos 1980. Estes processos encontram, no seu cerne, tanto modificações ao nível das organizações científicas, das práticas e princípios epistemológicos que estas encerram, 
como modificações ao nível dos contextos sociais onde estas organizações se inserem como ainda na relação estabelecida entre ambos . Apesar das especificidades destas modificações, da sua transversalidade e relevância relativa não serem, de todo, consensuais, a ciência é hoje entendida como resultante de colaborações científicas que se processam crescentemente a um nível transnacional e mobilizam tipos organizacionais diversos . Uma discussão mais aprofundada destas características do "fazer ciência" e do seu impacto na concretização investigação científica em Portugal serão os pontos centrais das próximas secções.

\section{Investigação científica: uma história de internacionalização crescente}

Focando-nos, num primeiro momento, na internacionalização da ciência, verificase que se os fluxos internacionais de pessoas e conhecimento existem há séculos, o seu crescimento extraordinário é fruto dos mais recentes processos de globalização, da facilidade de comunicação trazida pela utilização generalizada das tecnologias de informação e comunicação e, particularmente relevante para uma análise de caso que, como no estudo presente, tem por enfoque a investigação científica realizada, parcial ou totalmente em Portugal, das políticas comuns europeias .

Os indícios desta internacionalização da ciência têm sido revelados nas mais diversas áreas científicas e contextos nacionais . Portugal não é pois excepção. No contexto da ciência Portuguesa podem encontrar-se indicadores da internacionalização aos mais diversos níveis: já não só nas tradicionais cooperações científicas internacionais (projectos de investigação colaborativos; co-autoria de artigos científicos, constituição de redes de investigadores e mobilidade de cientistas) , mas igualmente, no desenvolvimento de actividades de investigação e desenvolvimento (I\&D) e de transferência de tecnologia no âmbito de organizações e consórcios internacionais envolvendo os sectores público e privado, na participação num mercado de trabalho científico e tecnológico internacional ou na concretização de programas de educação pós-graduada e de investigação envolvendo instituições de vários países . Estes processos de internacionalização foram estimulados por políticas de ciência que têm tido por objectivo o desenvolvimento do sistema científico e tecnológico nacional (num primeiro momento, através da Junta Nacional de Investigação Científica e Tecnológica (JNICT) e, mais recentemente, através da Fundação para a Ciência e a Tecnologia (FCT)) e têm sido o ponto central das políticas de ciência em Portugal desde a segunda metade dos anos 1990 . Esta 
promoção de um quadro de internacionalização crescente tem vindo a conduzir a uma convergência dos diversos sistemas científicos nacionais, apesar da variabilidade dos contextos nacionais onde as práticas científicas efectivamente se concretizam.

Factores que têm sido considerados para uma compreensão das especificidades nacionais da internacionalização da ciência passam, por exemplo, pelo estudo da dimensão de um país, que não sendo a única dimensão relevante nestes processos, tem sido reconhecida amplamente com tendo uma influência directa tanto na busca de conhecimento além-fronteiras como na mobilidade dos cientistas. Assim sendo, o caso português, é particularmente interessante de estudar. Trata-se de um pequeno país europeu, com uma comunidade científica que, apresentando um acentuado crescimento nas últimas décadas, permanece diminuta em relação à média europeia. Trata-se, igualmente, de uma comunidade que apresenta outputs científicos diminutos no contexto europeu e que apresenta uma das maiores taxas de colaborações internacionais em artigos científicos desde os anos 1980 . Outras dimensões previamente assinaladas como centrais para o desenvolvimento de redes internacionais de colaboração científica são as afinidades geográficas ou linguísticas, ligações históricas, culturais, económicas ou políticas . É assim que os países que mais têm colaborado com Portugal são Inglaterra (até ao início dos anos 2000) e Espanha (desde então). Portugal mantem ainda fortes ligações com os países de língua oficial Portuguesa, em particular com o Brasil . É neste quadro, que uma caracterização da investigação científica em malária terá necessariamente que abarcar o estudo da sua dimensão internacional. Área que permanece contudo por abordar.

\section{Os contextos organizacionais da investigação científica}

Paralelamente a esta dimensão da internacionalização do trabalho científico verifica-se ainda que a ciência é hoje fruto de crescentes colaborações entre tipos organizacionais diversos. Isto implica que à ciência concretizada através da aplicação do método científico, e durante muito tempo entendida como circunscrita no interior de um campo (semi) autónomo, teremos uma ciência que se revela, crescentemente, como um empreendimento heterogéneo resultante de constantes lutas, alianças e reposicionamentos de atores académicos, empresariais, (não)governamentais e da sociedade civil . Esta heterogeneidade organizacional, trazendo novos contributos ao "fazer ciência", nomeadamente um alargamento social da reflexividade científica, traduzir-se-á, simultaneamente, em decréscimos de autonomia do campo científico . 
Em Portugal, um panorama geral do desenvolvimento de actividades I\&D nos diferentes sectores de execução (empresas; organismos do Estado; ensino superior e instituições privadas sem fins lucrativos) ao longo do tempo tem sido efectivado através do Inquérito ao Potencial Científico e Tecnológico Nacional. Este inquérito é realizado regularmente em Portugal a partir de 1974 (uma síntese destes dados pode encontrar-se em ). No entanto, estudos científicos que tenham por enfoque os contextos organizacionais onde decorrem as práticas científicas, as relações estabelecidas entre contextos distintos e as transformações destas ligações ao longo do tempo, permanecem por concretizar. Apesar da inexistência destes estudos no contexto português, esta temática tem sido abordada noutros contextos nacionais e internacionais. Uma das primeiras investigações destas transformações das práticas científicas foi realizada por Hicks e Katz que mostraram que as publicações do Reino Unido apresentavam um perfil organizacional crescentemente diversificado entre 1981 e 1991 . Godin e Gingras, por seu lado, mostraram que entre 1980 e 1997 ocorreu uma diversificação dos contextos organizacionais onde decorrem as práticas científicas no Canadá. No entanto, estes autores mostraram ainda que a academia permaneceu no centro da produção científica, dado que a participação dos outros sectores de actividade revelou ser maioritariamente mediada pelas universidades . Num estudo centrado não num contexto nacional mas numa área científica (os estudos bibliométricos) verifica-se o mesmo tipo de padrão de diversificação organizacional a partir da década de 1990 . Em Portugal poder-se-ão encontrar algumas pistas relativas aos contextos organizacionais onde decorrem as práticas científicas em alguns trabalhos de investigação que abordam o papel das relações entre organizações diversas (empresas, universidade e outras) para o desenvolvimento de patentes ou actividades de inovação. Igualmente relevantes serão os estudos de caracter qualitativo que, não se centrando na caracterização dos contextos organizacionais onde decorrem as actividade I\&D, abordam, por exemplo, as motivações de actores empresariais e académicos para o estabelecimento de cooperações científicas . Finalmente encontram-se ainda em estudos de caso que partindo de organizações específicas, têm por enfoque práticas que envolvem tipos organizacionais diversos . Por abordar encontram-se ainda as transformações dos contextos organizacionais onde decorre a investigação científica em malária em Portugal ou noutro contexto geográfico. Sabe-se contudo que estas práticas decorrem em contextos organizacionais que não se cingem à academia . Permanece assim por realizar uma 
caracterização das configurações organizacionais da investigação científica em malária e as suas transformações ao longo do tempo. Este será pois um dos eixos do corrente trabalho.

É precisamente partindo deste quadro que este artigo centrar-se-á no mapeamento e caracterização exploratória da investigação científica em malária concretizada parcial ou totalmente em instituições Portuguesas entre 1900 e 2014. Esta caracterização basear-se-á no recurso a indicadores bibliométricos, uma abordagem recorrentemente mobilizada para aferir empiricamente as transformações na produção de conhecimento científico . Mais especificamente, mobilizar-se-ão todos os artigos científicos indexados num dos locus de legitimação máxima destas práticas: a Web of Science. Estas publicações traduzem assim a participação de instituições Portuguesas numa comunidade científica internacional. Nestas publicações, explorar-se-ão os padrões de evolução do número de publicações; o seu impacto; os autores e as instituições de pertença. Este trabalho pretende assim contribuir para uma caracterização de pontos centrais das várias teorias que têm, nas últimas décadas, pensado as transformações da ciência e assim aceder aos processos que conduziram à transformação da ciência produzida mais localmente e na ausência de diversidade organizacional, numa ciência que se concretiza crescentemente a uma escala global e em organizações diversas.

Partindo da revisão da literatura, a próxima secção elencará os objectivos desta investigação e as hipóteses de trabalho. Seguir-se-á a secção metodológica, onde se concretiza com maior precisão a abordagem empírica e técnicas mobilizadas, e a secção dos resultados, onde se apresentam os dados encontrados no decurso da investigação exploratória. $\mathrm{O}$ artigo termina com uma discussão dos resultados encontrados, das limitações do estudo presente e oportunidades para futuros trabalhos que complementem a investigação realizada. Um apontamento final releva os principais contributos do trabalho agora concretizado.

\section{Objectivos e hipóteses}

Este artigo tem por objectivo central estabelecer um mapa global do desenvolvimento da investigação científica em malária que é concretizada parcial ou totalmente em instituições Portuguesas e legitimada internacionalmente entre $1900 \mathrm{e}$ 2014. Paralelamente caracterizar-se-ão as transformações das práticas científicas no que concerne aos padrões de internacionalização e diversificação organizacional ao 
longo do período em análise.

Partindo da revisão da literatura, estabeleceram-se as seguintes hipóteses:

Hipótese 1. A investigação científica em malária que envolve instituições Portuguesas afirma-se, tanto quantitativa como qualitativamente, num plano internacional.

Esta hipótese decorre directamente de estudos que mostram que a investigação científica realizada em Portugal e analisada no seu conjunto apresenta, actualmente, padrões de internacionalização muito extensos . De notar que a internacionalização das práticas científicas não é um padrão exclusivo da ciência em Portugal, reflectindo, ao invés, tendências registadas ao nível da investigação científica em termos globais . Contudo, permanece por confirmar, primeiro se a investigação científica em malária apresenta um padrão de internacionalização similar (de notar que as diferentes áreas científicas apresentam padrões de internacionalização diferenciados ), e segundo, se esta investigação, mais do que participar em redes de colaboração internacional, se revela como um actor relevante no plano internacional.

Esta hipótese será confirmada ou infirmada recorrendo a vários tipos de análise que se passarão a elencar. Primeiro, analisar-se-á a variação do número de artigos indexados na Web of Science entre 1900 e 2014 e, em particular, estabelecer-se-á uma comparação entre os artigos exclusivamente concretizados em instituições Portuguesas (artigos nacionais) e aqueles que são fruto de colaborações entre instituições Portuguesas e instituições de outros países (artigos internacionais). De acordo com a revisão da literatura e a hipótese estabelecida, o número de artigos indexados, tanto nacionais como internacionais, terá sofrido um crescimento acelerado nas últimas décadas.

Segundo, reconhecendo que o factor de impacto das revistas científicas, apesar de todas as suas insuficiências e problemas, é considerado, no campo das ciências da saúde, um indicador válido da qualidade das revistas científicas, caracterizar-se-á a evolução do impacto da investigação em malária entre 1900 e 2014 e comparar-se-ão os impactos das publicações exclusivamente Portuguesas e daquelas que são fruto de trabalho colaborativo entre instituições Portuguesas e instituições de outros países. De acordo com a hipótese estabelecida, o impacto da investigação em malária terá crescido ao longo do tempo apresentando as publicações em colaboração internacional um maior impacto do que as publicações exclusivamente Portuguesas. Esta asserção (de um maior impacto de publicações envolvendo parceiros de vários 
países por comparação ao impacto de publicações exclusivamente nacionais), não sendo transversal a todas as áreas científicas, foi previamente estabelecida para a área das ciências biomédicas em publicações indexadas de 50 países .

Finalmente, reconhecendo que o posicionamento na lista de autores é, no campo das ciências da saúde, revelador da contribuição de cada cientista para o trabalho efectuado , verificar-se-ão os padrões de posicionamento dos autores de instituições Portuguesas nas publicações indexadas na Web of Science. Mais especificamente, o autor cujo nome surge em primeiro lugar é o principal responsável pela execução empírica do trabalho de investigação. $\mathrm{O}$ autor que surge em último lugar é o investigador responsável pelo projecto de investigação. Os autores com posicionamentos intermédios realizaram contributos mais pequenos na prossecução do artigo em causa. Assim sendo, se um acréscimo de primeiras autorias com afiliações Portuguesas revelaria a concretização da maioria do trabalho empírico em Portugal, um crescimento de últimas autorias com afiliações institucionais de Portugal, indicaria um crescimento da independência científica da investigação realizada por investigadores a trabalhar em Portugal e a afirmação científica dos mesmos no plano internacional. Uma afirmação crescente num plano internacional deverá ser revelada tanto por um crescimento das primeiras como das últimas autorias nas publicações indexadas na Web of Science.

Hipótese 2. O padrão de crescente heterogeneidade organizacional não é independente da crescente internacionalização da investigação científica em malária.

Tal como referido anteriormente (ver Secção 3), a ciência que no início da modernidade se encontrava maioritariamente encerrada no interior de um campo científico relativamente autónomo, tem vindo a transformar-se numa prática que envolve os mais diversos tipos de actores: desde a academia, à sociedade civil, passando por organismos estatais (laboratórios de Estado; ministérios; outros) e museus, desde organizações não-governamentais a hospitais entre outros. Tal como proposto e descrito nas mais diversas publicações, a ciência seria assim hoje caracterizada por uma heterogeneidade institucional (revisto em ). Esta crescente diversidade organizacional foi aferida na investigação científica concretizada em contextos nacionais e temáticos distintos dos que serão aqui abordados . Um primeiro contributo será pois avaliar os padrões de desenvolvimento dos contextos organizacionais onde decorrem estas práticas. 
Ainda de acordo com a hipótese acima elencada - hipótese 2 - este modelo heterogéneo de fazer ciência, seria, no caso da investigação em malária concretizada em Portugal importado do exterior, revelando-se mais proeminente nas investigações concretizadas em colaboração internacional do que nos trabalhos exclusivamente nacionais. Esta hipótese, que advém da centralidade da internacionalização da investigação científica para o desenvolvimento da ciência e tecnologia em Portugal, internacionalização esta continuamente promovida por iniciativas do foro político, institucionais e pessoais, permanece por explorar tanto no contexto genérico do fazer ciência em Portugal, como no contexto mais específico da investigação em malária concretizada em instituições Portuguesas.

\section{Métodos}

Num momento inicial, procedeu-se à identificação e recolha exaustiva e sistemática dos artigos científicos que 1) contêm nos seus títulos e/ou resumos as palavras "Malaria" e/ou "Plasmodium"; 2) foram publicados entre o início de 1900 e o final de 2014 ; 3) apresentam um ou vários autores afiliados em instituições Portuguesas e 4) encontram-se indexados na Web of Science, uma base de dados privada (Thomson Reuters) que sistematiza publicações científicas datadas a partir de 1900. A recolha foi efectuada entre Janeiro e Março 2015 na plataforma http://apps.webofknowledge.com/.

De acordo com estes critérios seleccionaram-se 472 artigos, entre os quais encontravam-se 5 artigos mal classificados (1 artigo que não se debruçava sobre malária e 4 que não apresentavam autores afiliados em instituições Portuguesas). Entre os 467 artigos que compõem o universo, procedeu-se, num segundo momento, à caracterização dos seguintes tópicos: ano de publicação; país de afiliação dos autores (exclusivamente Portugal - denominado como "artigo nacional", ou incluindo afiliações de Portugal e outro(s) país(es) - artigo classificado como "artigo internacional"); factor de impacto do Journal Citation Reports (Web of Science) de 2014 a 1 ano e a 5 anos (por forma a simplificar a análise os factores de impacto, estabeleceram-se os seguintes agrupamentos: inferior a 2.0; entre $2.0 \mathrm{e}$ 10.0; superior a 10.0); posicionamento nas autorias dos cientistas afiliados em instituições Portuguesas (primeiro autor; último autor); tipos organizacionais (presença de um tipo organizacional; dois tipos organizacionais; três ou mais tipos organizacionais). 
Recorreu-se ao teste de Qui-quadrado para avaliar a independência de variáveis nominais. Os resíduos estandardizados ajustados foram utilizados para avaliar associações entre categorias de variáveis nominais. Para variáveis ordinais, o teste de Mann-Whitney foi mobilizado para testar diferenças significativas entre grupos de artigos.

A análise estatística foi realizada com o programa IBM SPSS Statistics, version 20.

\section{Resultados}

\subsection{Caracterização dos artigos publicados em revistas indexadas entre 1900 e 2014}

Uma primeira análise dos artigos publicados entre 1900 e 2014 e indexados na Web of Science indica a publicação de apenas um artigo em 1900 seguido de uma longa interrupção até 1979. Este padrão será possivelmente reflexo dos padrões de investigação científica em Portugal nos primeiros três quartos do século XX. A investigação em malária em Portugal à época seria direccionada para um público falante do idioma português e fora dos fora internacionais hoje considerados como de legitimação máxima das práticas científicas em biomedicina. De facto, o número de publicações só começa a crescer consistentemente na última década do século XX, encontrando-se $98.5 \%$ destas publicações em datas posteriores a 1995 (Figura 1A). Na verdade, o padrão de desenvolvimento de artigos assinados por cientistas a trabalhar em instituições Portuguesas é consistente com o desenvolvimento do sistema científico e tecnológico em Portugal e com o acentuado investimento no seu desenvolvimento nomeadamente através de políticas de apoio à internacionalização. Recorrendo à análise das publicações indexadas na Web of Science, encontra-se aqui reflectido o desenvolvimento do sistema científico e tenológico nacional, num primeiro momento, através da JNICT e, mais acentuadamente, a partir da segunda metade dos anos 90 através da FCT .

Os dados revelam ainda que tanto o número total de artigos (onde participam instituições Portuguesas), como o número de artigos nacionais (de autoria exclusiva de instituições Portuguesas) e o número de artigos internacionais (onde instituições Portuguesas colaboram com instituições de outros países) apresentam um crescimento acentuado ao longo do tempo. 
Figura 1. Artigos científicos em malária indexados na Web of Science.
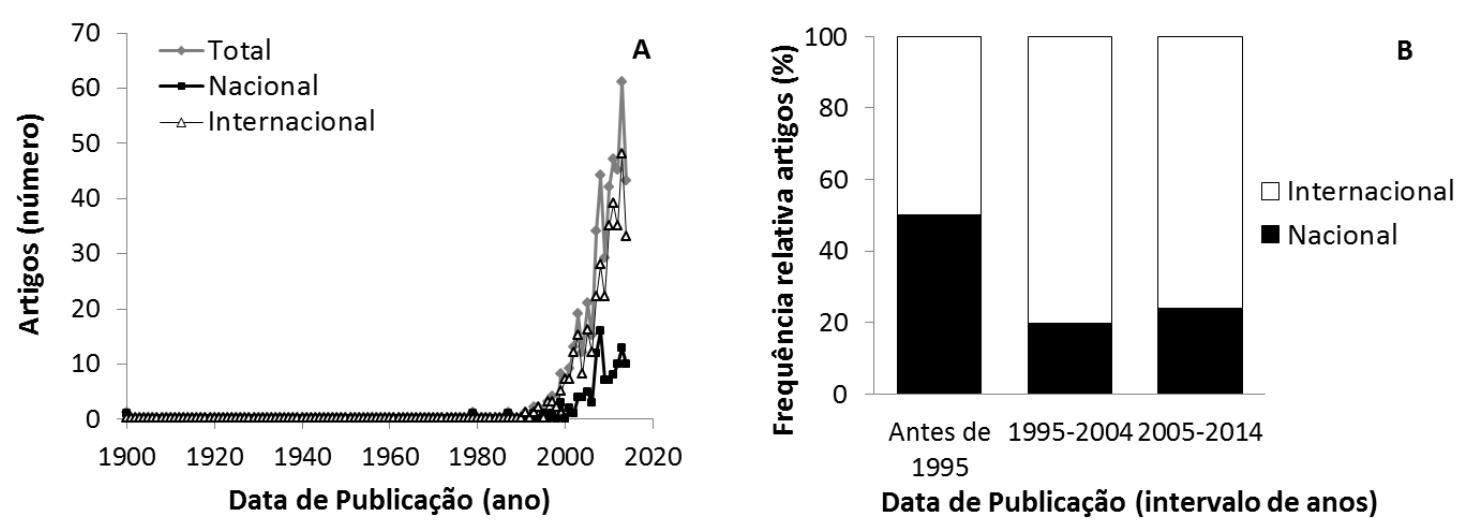

Figura 1A. Número de artigos publicados entre 1900 e 2014. Os dados são expressos como número total de artigos (cinzento); número de artigos nacionais (preto) e número de artigos internacionais (branco).

Figura 1 B. Frequências relativas de artigos nacionais e internacionais. Os dados são expressos como frequências relativas de artigos nacionais (preto) e internacionais (branco) antes de 1995 (à esquerda no gráfico); entre 1995 e 2004 (ao centro do gráfico) e entre 2005 e 2014 (à direita no gráfico).

Os dados revelam ainda que o crescimento do número total de artigos internacionais é superior ao crescimento do número total de artigos nacionais (Figura 1A). Este padrão é mais facilmente discernível através da comparação das frequências relativas de artigos nacionais com as frequências relativas de artigos internacionais (Figura 1B). Globalmente observa-se um claro favorecimento de publicações em colaboração internacional (76.2\% de todas as publicações analisadas) verificando-se ainda que as publicações internacionais apresentam um crescimento relativo intenso até aos primeiros anos do século XXI, encontrando-se este crescimento estável desde então (Figura 1B). Acresce, que a percentagem de artigos nacionais é significativamente inferior à percentagem de artigos internacionais a partir de 1995, o que é revelador de um padrão acentuado de internacionalização da investigação em malária.

Uma forma complementar de analisar o impacto relativo das publicações em malária poderá realizar-se através de uma análise dos factores de impacto das revistas (a 1 ano e a 5 anos). Esta análise revela, num primeiro momento, um crescimento dos factores de impacto das publicações em malária ao longo do período analisado. Este crescimento encontra-se patente tanto nas publicações nacionais como nas internacionais ${ }^{2}$. Acresce

\footnotetext{
2 Analisando os artigos de uma forma agregada verifica-se que no período anterior a 1995 o factor de impacto médio é de 2.2; no período entre 1995 e 2004 o factor de impacto situa-se em 3.8 e no período entre 2005 e 2014 em 4.6. A mesma tendência global de crescimento está presente numa análise desagregada em artigos nacionais e internacionais $(1.8 ; 3.2 ; 3.8$ e $2.2 ; 4.3 ; 5.0$, respectivamente).
} 
que os factores de impacto das publicações analisadas não são independentes da natureza nacional ou internacional dos artigos científicos $\left(\chi_{(2)}^{2}=20.953\right.$; $p<0.001$ e $\chi_{(2)}^{2}=$ 17.876; $\mathrm{p}<0.001$, para factores de impacto a 1 e 5 anos, respectivamente) ${ }^{3}$. Mais especificamente, os dados revelam que enquanto as publicações nacionais apresentam uma maior probabilidade de serem concretizadas em revistas de maior impacto, as publicações internacionais apresentam uma maior probabilidade de se materializarem em revistas científicos com impacto superior (entre 2.0 e 10.0), mas não nas revistas reconhecidas com as de legitimidade máxima (com factores de impacto iguais ou superiores a 10.0$)^{4}$ (Tabela 1$)$.

Esta análise aponta, por um lado, para uma afirmação crescente da ciência produzida em instituições Portuguesas, mas por outro, e em consonância com estudos internacionais anteriores ao presente trabalho, para uma maior afirmação da ciência produzida em redes de colaboração internacional.

Tabela 1. Factores de impacto em artigos nacionais e internacionais

\begin{tabular}{|c|c|c|c|c|}
\hline & & Nacionais & Internacionais & Total \\
\hline Factor de impacto JCR & Inferior a 2.0 & $4.5 *(32.7 \%)$ & $-4.5^{*}(13.1 \%)$ & $17.5 \%$ \\
\hline \multirow[t]{3}{*}{2014 (1 ano) } & Entre 2.0 e 10.0 & $-4.1^{*}$ & 4.1* $(80.9 \%)$ & $76.5 \%$ \\
\hline & Igual ou superior a & $(61.4 \%)$ & $0.0(6.0 \%)$ & $6.0 \%$ \\
\hline & 10.0 & $0.0(5.9 \%)$ & & \\
\hline Factor de impacto JCR & Inferior a 2.0 & $4.2 *(23.5 \%)$ & $-4.2 *(8.1 \%)$ & $11.5 \%$ \\
\hline 2014 & Entre 2.0 e 10.0 & $-3.1 *$ & 3.1* $(80.9 \%)$ & $81.1 \%$ \\
\hline \multirow[t]{2}{*}{ (5 anos) } & Igual ou superior a & $(61.4 \%)$ & $0.6(7.8 \%)$ & $7.4 \%$ \\
\hline & 10.0 & $-0.6(6.1 \%)$ & & \\
\hline
\end{tabular}

Nota: Valores expressos com resíduos estandardizados ajustados e percentagens de publicações nacionais e internacionais. * Indica valores estatisticamente significativos $(|Z|>1.96$; nível de significância de 0.05); negrito indica probabilidades de associação significativas e positivas.

JCR: Journal Citation Reports

\footnotetext{
3 Uma análise complementar à apresentada no texto confirma os resultados expostos. Efectivamente, os factores de impacto das publicações internacionais (aqui considerada como variável métrica) são significativamente superiores aos das publicações nacionais (Mann-Whitney $\mathrm{U}=12630.0, p<0.001$; MannWhitney $\mathrm{U}=11964.5, p<0.001$, para a análise dos factores de impacto a 1 e 5 anos, respectivamente).

${ }^{4} \mathrm{~A}$ análise do impacto das publicações, por hora cingida à análise de factores de impacto, será futuramente completada por uma análise do número de citações de cada artigo, acedendo assim, de uma forma mais directa, ainda que não sem problemas, ao impacto de cada artigo do universo analisado (e não a um impacto conjunto da revista onde os artigos foram publicados).
} 
Finalmente, recorrendo a um dos modos mais comummente utilizados para a análise do investimento na internacionalização da ciência, caracterizar-se-á de seguida o padrão de colaborações internacionais, aferido através das co-autorias de artigos científicos .

Os dados expressos na Figura $2 A$ revelam um crescimento do número total de artigos que apresenta autores afiliados em instituições Portuguesas tanto como primeiro como como últimos autores. Adicionalmente, verifica-se que se entre $1900 \mathrm{e}$ 2014 ocorreu efectivamente um crescimento acentuado das frequências relativas de primeiras autorias nacionais, o mesmo não se verifica ao nível últimas autorias nacionais, que se mantiveram relativamente estáveis no decorrer do período de tempo analisado (Figura 2B). Estes dados revelam que apesar da número de publicações ser muito reduzido no período que antecedeu 1995, a independência relativa da investigação concretizada por autores Portugueses é similar à da actualidade. Assim, apesar do crescimento acentuado das publicações e do seu impacto, a investigação concretizada em Portugal não parece apresentar uma autonomia crescente. Conjuntamente, estes dados revelam que se a independência científica da investigação em malária se manteve estável em termos relativos, uma parte muito relevante da concretização empírica destas investigações passa, em grande parte, pelas instituições Portuguesas.

Em último lugar, uma análise mais fina das publicações internacionais revela que cerca de metade destas publicações inscrevem uma autonomia relativa das organizações científicas Portuguesas (Figura 2C). Neste grupo incluem-se os artigos com últimas autorias de organizações Portuguesas - onde Portugal foi capaz de captar investigadores de organizações de outros países para a concretização empírica dos seus projectos; e primeiras e últimas autorias de cientistas a trabalhar em Portugal nos quais possivelmente se delegou uma pequena parte do trabalho a investigadores de organizações não Portuguesas. Na segunda metade dos artigos internacionais, os investigadores a trabalhar em instituições Portuguesas apresentam uma posição subalterna. Em cerca de $33 \%$ destes artigos estes investigadores desempenham funções menores (autorias intermédias) e nos restantes $15 \%$, concretizam a maior parte do trabalho empírico, tendo assim ficado dotados do conhecimento prático do trabalho de investigação, conhecimento esse que os capacita para uma futura concretização científica autónoma (potencial de apresentarem últimas autorias no futuro). 


\section{Figura 2. Número de artigos científicos indexados na Web of Science com} autorias nacionais.

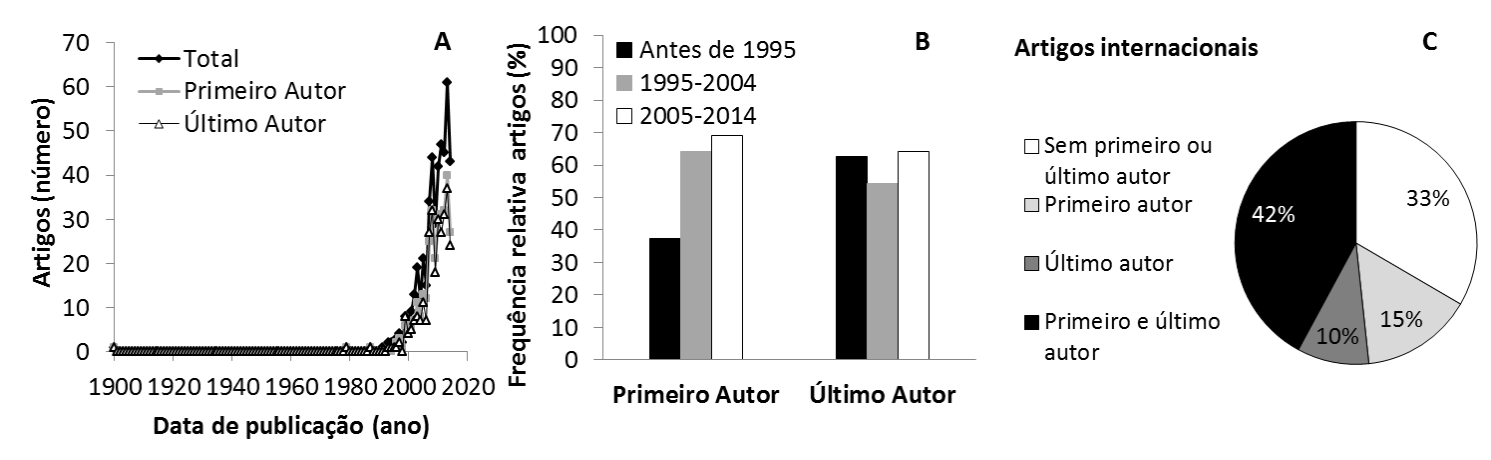

Figura 2A. Número de artigos publicados entre 1900 e 2014 por tipo de autoria nacional. Os dados são expressos como número total de artigos (preto); número de artigos com primeiro autor nacional (cinzento) e número de artigos com último autor nacional (branco). Figura $2 B$. Frequências relativas de artigos com primeiro e último autor nacional. Os dados são expressos como frequências relativas de artigos com primeiro autor (à esquerda no gráfico) ou último autor nacional (à direita no gráfico) antes de 1995 (preto); entre 1995 e 2004 (cinzento) e entre 2005 e 2014 (branco). Figura 2C. Frequências relativas de artigos internacionais. Os dados são expressos como frequências relativas de artigos sem primeiro ou último autor nacional (branco); com primeiro autor nacional (cinzento claro); com último autor nacional (cinzento escuro); com primeiro e último autor nacional (preto).

No seu conjunto, os dados apresentados confirmam a hipótese 1: a investigação em malária que envolve instituições Portuguesas afirma-se tanto quantitativamente como qualitativamente num plano internacional (crescente número de artigos; crescentes factores de impacto e crescentes primeiras e últimas autorias de publicações indexadas na Web of Science). Ainda assim, estas publicações permanecem a apresentar um padrão de subalternidade às organizações científicas internacionais (o peso relativo das publicações em colaboração internacional é três vezes superior ao das publicações nacionais; os factores de impacto das publicações internacionais são significativamente maiores que os das publicações nacionais; há uma estabilidade do peso relativo dos artigos com últimas autorias nacionais e cerca de metade das publicações internacionais não são lideradas por cientistas a trabalhar em organizações Portuguesas). 


\subsection{Os contextos organizacionais}

Os contextos organizacionais onde as publicações analisadas foram realizadas são diversificados, destacando-se, como seria expectável, as universidades ou institutos de investigação (presentes em $96.1 \%$ de todas as publicações); os hospitais (34.0\%) e os organismos do estado (28.1\%). Estes dados confirmam estudos prévios que apesar de revelarem uma diversidade organizacional crescente ao longo do tempo, afirmam também a centralidade da academia na concretização dos artigos científicos .Uma análise mais fina da evolução dos contextos organizacionais onde decorrem estas práticas científicas indica que, tal como para as restantes análises efectuadas (Figuras $1 A$ e 2A), o número total de publicações que apresentam um único tipo organizacional, dois tipos organizacionais ou três ou mais tipos organizacionais é crescente (Figura 3A). Adicionalmente, as frequências relativas de artigos onde é privilegiada uma homogeneidade organizacional (um único tipo organizacional) ou onde coexistem dois tipos organizacionais são relativamente estáveis (Figura 3B). Pelo contrário, a frequência relativa das publicações com 3 ou mais tipos organizacionais apresenta-se em crescendo no período analisado.

\section{Figura 3. Contextos Organizacionais dos artigos científicos em malária indexados}

\section{na Web of Science.}
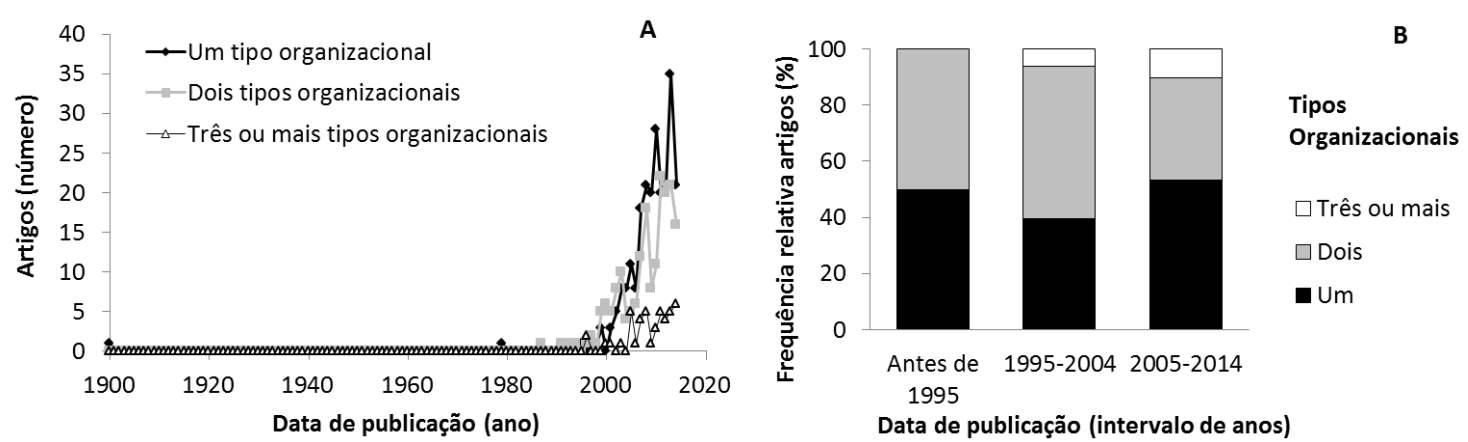

Figura 3A. Número de artigos publicados entre 1900 e 2014 por tipos organizacionais. Os dados são expressos como número total de artigos com um tipo organizacional (preto); com dois tipos organizacionais (cinzento) e com três ou mais tipos organizacionais (branco). Figura 3B. Frequências relativas de artigos com um ou mais tipos organizacionais. Os dados são expressos como frequências relativas de artigos com um tipo organizacional (preto), dois tipos organizacionais (cinzento) ou três ou mais tipos organizacionais (branco) antes de 1995 (à esquerda no gráfico); entre 1995 e 2004 (ao centro do gráfico) e entre 2005 e 2014 (à direita no gráfico). 
Finalmente avaliou-se se a diversidade organizacional seria independente da origem nacional ou internacional das autorias. Confirmando a hipótese 2, a análise do qui quadrado revela que a diversidade organizacional não é independente da natureza nacional/internacional das autorias $\left(\chi_{(2)}^{2}=57.314 ; p<0.001\right)^{5}$. Uma análise mais detalhada destes dados indica que os artigos nacionais apresentam uma maior probabilidade de apenas apresentarem um único tipo organizacional (Tabela 2). Por oposição, os artigos internacionais revelam uma maior probabilidade de terem como autores dois ou mais tipos organizacionais.

Tabela 2. Tipos organizacionais de artigos nacionais e internacionais

\begin{tabular}{lllll}
\hline & & Nacionais & Internacionais & Total \\
\hline Tipos Organizacionais & Um & $\mathbf{7 . 5}^{*}(82.0 \%)$ & $-7.5^{*}(41.3 \%)$ & $51.0 \%$ \\
& Dois & $-5.6^{*}(17.1 \%)$ & $\mathbf{5 . 6}^{*}(46.6 \%)$ & $39.6 \%$ \\
& Três ou mais & $-3.5(0.9 \%)$ & $\mathbf{3 . 5}^{*}(12.1 \%)$ & $9.4 \%$ \\
\hline
\end{tabular}

Nota: Valores expressos com resíduos estandardizados ajustados e percentagens de publicações nacionais e internacionais. * Indica valores estatisticamente significativos ( $|Z|>1.96$; nível de significância de 0.05); negrito indica probabilidades de associação positivas significativas.

No seu conjunto, os dados apontam para um alargamento dos contextos organizacionais onde decorrem estas práticas científicas, concretizando-se um modelo de fazer ciência que aparenta ser replicado de outras geografias.

\section{Discussão}

Este trabalho tem como principal contributo um primeiro mapeamento e caracterização da investigação científica em malária realizada parcial ou totalmente em instituições Portuguesas desde o início do século XX. A análise exploratória realizada revela primeiro, um crescimento muito acentuado desta investigação após 1995 e segundo, que esta investigação se caracteriza, simultaneamente, por padrões de internacionalização e heterogeneização organizacional crescente. Confirmaram-se

\footnotetext{
5 Uma análise complementar à apresentada no texto confirma os dados apresentados: a diversidade organizacional é significativamente diferente entre artigos nacionais e internacioains (Mann-Whitney U $=11393.0, p<0.001$ ), revelando uma maior probabilidade dos artigos internacionais serem dotados de maior heterogeneidade organizacional.
} 
assim, para o caso particular da investigação científica em malária, que as práticas científicas se têm modificado ao longo do período de tempo analisado e de uma forma mais evidente e em consonância com o desenvolvimento do sistema científico e tecnológico nacional, após 1995. Estas modificações não só conjugam as anteriormente reportadas tendências de internacionalização e diversificação dos contextos organizacionais, mas acrescentaram a estes modelos uma relação entre a diversificação organizacional e os padrões de internacionalização de ciência. No futuro seria interessante questionar os impactos destes processos na ciência efectivamente produzida. Se por um lado estas características poderão permitir aceder a conhecimentos e competências inexistentes, ou pouco preponderantes, no contexto nacional ou no contexto da academia, por outro, a questão que se pode colocar agora é em que medida estes padrões enquadram a base científica da investigação per se: as questões que são colocadas, as metodologias e técnicas mobilizadas e, consequentemente, os resultados alcançados e conclusões propostas. Uma vez caracterizados os locus concretos das práticas científicas e processos que subjazem às modificações encontradas, poder-se-á agora aceder à base científica da investigação desenvolvida, aferindo se esta se encontra, tal como os seus locus e actores, enquadrada pelas transformações que as práticas científicas revelam, mais acentuadamente, desde a década de 1980.

Apesar dos contributos aqui sublinhados, a análise realizada apresenta algumas limitações que serão agora discutidas. Em primeiro lugar refere-se a mobilização da Web of Science como o ponto de partida para a análise das publicações em malária que envolvem organizações Portuguesas. Se estas bases de dados são, no contexto das ciências da saúde, incontornáveis nas últimas décadas, o mesmo não se verificaria no início do século XX. Neste mesmo sentido, as publicações em malária em Portugal, gozavam nessa época de outros fora de legitimação como A Medicina Contemporanea ou os Anais do Instituto de Medicina Tropical. Apenas a análise destas e de outras publicações similares permitiria que nos aproximássemos de um quadro mais completo da investigação efectivamente realizada em Portugal.

Uma segunda limitação advém desta análise cingir-se à ciência concretizada, parcial ou totalmente, em organizações Portuguesas. Trata-se então de um estudo de caso, que sendo valioso pelas pistas que nos faculta no que concerne à ciência produzida em Portugal, não permite aferir a importância relativa deste contributo num contexto mais amplo. Esta comparação permanece assim por realizar. Pode referir-se 
ainda que sendo o enfoque deste estudo a investigação concretizada numa doença com características biológicas e impactos sociais específicos - trata-se de uma patologia infecciosa que, colocando em risco $45 \%$ da população mundial, afecta maioritariamente países pobres e populações carenciadas e cujos expectáveis lucros financeiros para a indústria farmacêutica são muito reduzidos ou nulos - o alargamento da presente caracterização à investigação científica, mesmo no interior das ciências da saúde, à investigação científica de outras patologias não poderá ser realizado sem mais. Futuramente seria interessante recorrer ao mesmo tipo de abordagem empírica para a caracterização do estudo de patologias com enquadramentos diversos.

Finalmente, a caracterização aqui concretizada poderá ser futuramente alargada a características que, tal como a internacionalização a diversificação dos contextos organizacionais, têm sido associadas às transformações das práticas científicas mas que têm sido menos consensuais nas análises realizadas. Aferir-se-á por exemplo a presença crescente de uma "ciência aplicada" ou a mobilização de quadros teóricos e abordagens metodológicas interdisciplinares ou transdisciplinares (uma comparação dos diversos quadros teóricos das transformações de ciência pode encontrar-se em ). Este será o próximo passo no desenvolvimento do estudo exploratório agora apresentado.

Apesar das limitações do presente trabalho, conseguiu-se estabelecer um primeiro mapa da investigação em malária concretizada por instituições Portuguesas. Esta abordagem revelou que o crescimento desta investigação é dominado por um modelo de internacionalização de ciência que tem vindo a promover crescentes conexões entre os domínios académico, político, económico e social.

\section{Agradecimentos}

Ana Ferreira tem uma bolsa de Pós-doutoramento da Fundação para a Ciência e a Tecnologia (SFRH/BPD/77611/2011). A autora agradece o contributo crítico de Ana Lúcia Teixeira na discussão e revisão dos procedimentos estatísticos.

\section{Referências}

Böhme, Gernot, Wolfgang Van den Daele, Rainer Hohlfeld, Wolf Krohn, and Wolf Schäfer. Finalization in Science: The Social Orientation of Scientific Progress. edited by Wolf Schäfer Dordrecht: D. Reidel Publishing Company, 1983. 
Bozeman, Barry, Daniel Fay, and Catherine P. Slade. "Research Collaboration in Universities and Academic Entrepreneurship: The-State-of-the-Art." The Journal of Technology Transfer 38, no. 1 (2012): 1-67.

Bruce-Chwatt, L. J., and Julian de Zulueta. "Malaria Eradication in Portugal." Transactions of the Royal Society of Tropical Medicine and Hygiene 71, no. 3 (1977/01/01 1977): 232-40.

Coccia, Mario, and Lili Wang. "Evolution and Convergence of the Patterns of International Scientific Collaboration." Proceedings of the National Academy of Sciences 113, no. 8 (February 23, 2016 2016): 2057-61.

Daems, Rutger, Edith Maes, Maneesha Mehra, Benjamin Carroll, and Adrian Thomas. "Pharmaceutical Portfolio Management: Global Disease Burden and Corporate Performance Metrics." Value in Health 17, no. 6 (9// 2014): 732-38.

Dance, Amber. "Who's on First?". Nature 489, no. 7417 (2012): 591-92.

Delicado, Ana. "Cientistas Portugueses No Estrangeiro: Factores De Mobilidade E Relações De Diáspora." Sociologia Problemas e Práticas 58, no. 109-129 (2008).

—_. "O Retorno Dos "Cérebros": Regresso E Reintegração Dos Investigadores Portugueses Em Mobilidade." Revista iberoamericana de ciencia tecnología y sociedad 5, no. 15 (2010): 185-218.

Edquist, Charles. Systems of Innovation: Technologies, Institutions and Organizations. London: Pinter Publishers/Cassell Academic, 1997.

Etzkowitz, Henry, and Loet Leydesdorff. "The Endless Transition: A "Triple Helix" of University-Industry-Government Relations." [In English]. Minerva 36, no. 3 (1998/09/01 1998): 203-08.

Faustino, Vítor. "Controlo Populacional E Erradicação Da Malária: O Caso Dos Ranchos Migratórios." In A Circulação Do Conhecimento: Medicina, Redes E Impérios, edited by Cristiana Bastos, 435-56. Lisboa: ICS - Imprensa de Ciências Sociais, 2011.

Fernandes, Lino. "Políticas De Dinamização De I\&D Empresarial." In 40 Anos De Políticas De Ciências E De Ensino Superior, 235-63. Lisboa: Almedina, 2015. Fonseca, Ana Glória, Sara S. Dias, Joao Luis Baptista, and Jorge Torgal. "The Burden of Imported Malaria in Portugal 2003 to 2012." Journal of Travel Medicine 21, no. 5 (2014): $354-56$. 
Fontes, Margarida, and Emília Araújo. "(I)Mobilidades E Redes Científicas Internacionais: Contextos E Relações Em Mudança." In Para um Debate sobre Mobilidade e Fuga de Cérebros, edited by Emília Araújo, Margarida Fontes and Sofia Bento Braga: Centro de Estudos de Comunicação e Sociedade, Universidade do Minho, 2013.

Fontes, Margarida, Pedro Videira, and Teresa Calapez. "The Impact of Long-Term Scientific Mobility on the Creation of Persistent Knowledge Networks." Mobilities 8, no. 3 (2013): 440-65.

Frame, J. Davidson , and Mark P. Carpenter. "International Research Collaboration." Social Studies of Science 9, no. 4 (November 1, 1979 1979): 481-97.

Funtowicz, Silvio O., and Jerome R. Ravetz. "Science for the Post-Normal Age." Futures 25, no. 7 (9// 1993): 739-55.

Garcia, José Luís, and Hermínio Martins. "O Ethos Da Ciência E Suas Transformações Contemporâneas, Com Especial Atenção À Biotecnologia." Scientiae Studia 7 (2009): 83-104.

Gibbons, Michael, Camille Limoges, Helga Nowotny, Simon Schwartzman, Peter Scott, and Martin Trow. The New Production of Knowledge: The Dynamics of Science and Research in Contemporary Societies. SAGE Publications, 1994.

Glänzel, Wolfgang. "National Characteristics in International Scientific CoAuthorship Relations." Scientometrics 51, no. 1 (2001): 69-115.

Godin, Benoit, and Yves Gingras. "The Place of Universities in the System of Knowledge Production." Research Policy 29, no. 2 (2// 2000): 273-78.

Heitor, Manuel. "Ciência E Conhecimento Na Modernização De Portugal." In 40 Anos De Políticas De Ciências E De Ensino Superior, 81-146. Lisboa: Almedina, 2015.

Hessels, Laurens K., and Harro van Lente. "Re-Thinking New Knowledge Production: A Literature Review and a Research Agenda." Research Policy 37, no. 4 (5// 2008): 740-60.

Hicks, Diana M., and J. Sylvan Katz. "Where Is Science Going?". Science, Technology \& Human Values 21, no. 4 (October 1, 1996 1996): 379-406.

Horta, Hugo, and Maria Teresa Patrício. "Setting-up an International Science Partnership Program: A Case Study between Portuguese and Us Research Universities." Technological Forecasting and Social Change In press (2015). 
Irvine, John, and Ben R. Martin. Foresight in Science: Picking the Winners. Pinter, 1984.

Lezaun, Javier, and Catherine M. Montgomery. "The Pharmaceutical Commons: Sharing and Exclusion in Global Health Drug Development." Science, Technology \& Human Values 40, no. 1 (January 1, 2015 2015): 3-29.

Lobo, Ana Rita Merelo. "A História Da Malária Em Portugal Na Transição Do Século Xix Para O Século Xx E a Contribuição Da Escola De Medicina Tropical De Lisboa (1902-1935)." FCT-UNL, 2012.

Luukkonen, Terttu, Olle Persson, and Gunnar Sivertsen. "Understanding Patterns of International Scientific Collaboration." Science, Technology \& Human Values 17, no. 1 (January 1, 1992 1992): 101-26.

Marques, João P. C., João M. G. Caraça, and Henrique Diz. "How Can UniversityIndustry-Government Interactions Change the Innovation Scenario in Portugal? - the Case of the University of Coimbra." Technovation 26, no. 4 (2006): 534-42.

Martin, Ben R. "What Can Bibliometrics Tell Us About Changes in the Mode of Knowledge Production?". Prometheus 29, no. 4 (2011/12/01 2011): 455-79.

Mendonça, Joana. "Observação E Conhecimento: Cinco Décadas De Indidadores C\&T." In 40 Anos De Políticas De Ciências E De Ensino Superior, 395-426. Lisboa: Almedina, 2015.

Moore, Kelly, Daniel Lee Kleinman, David Hess, and Scott Frickel. "Science and Neoliberal Globalization: A Political Sociological Approach." [In English]. Theory and Society 40, no. 5 (2011/09/01 2011): 505-32.

Moutinho, Paula Susana Figueiredo, Margarida Fontes, and Manuel Mira Godinho. "Do Individual Factors Matter? A Survey of Scientists' Patenting in Portuguese Public Research Organisations." Scientometrics 70, no. 2 (2007): 355-77.

Nowotny, H., P. Scott, and M.T. Gibbons. Re-Thinking Science: Knowledge and the Public in an Age of Uncertainty. Wiley, 2001.

Oliveira, Luísa, and Helena Carvalho. "Why Firms Do Not Enrol in Socio-Technical Networks - Empirical Evidence from Portugal." Sociology of Science and Technology, Institute for the History of Science and Technology/Russian Academy of Sciences 1, no. 3 (2010): 73-84. 
Patrício, Maria Teresa. "Science Policy and the Internationalisation of Research in Portugal ". Journal of Studies in International Education 14 (2011): 161-82.

Patrício, Maria Teresa, and Tiago Santos Pereira. "A Política Científica Europeia E a Internacionalização Da Ciência." In 40 Anos De Políticas De Ciência E De Ensino Superior, edited by Maria de Lurdes Rodrigues and Manuel Heitor, 221-43. Coimbra: Edições Almedina, 2015.

Pinheiro, Miguel Linhares, José Carlos Pinho, and Cândida Lucas. "The Outset of U-I R \& D Relationships: The Specific Case of Biological Sciences." European Journal of Innovation Management 18, no. 3 (2015): 282-306.

Pollock, Anne. "Places of Pharmaceutical Knowledge-Making: Global Health, Postcolonial Science, and Hope in South African Drug Discovery." Social Studies of Science 44, no. 6 (December 1, 2014 2014): 848-73.

Saavedra, Mónica. A Malária Em Portugal: Histórias E Memórias Lisboa: ICS, 2014. "Malária, Mosquitos E Ruralidade No Portugal Do Século Xx ". Etnográfica 17, no. 1 (2013): 51-76.

. "Mosquitos Envenenados: Arrozais E Malaria Em Portugal." In A Circulação Do Conhecimento: Medicina, Redes E Impérios. , edited by Cristiana Bastos, 417-34. Lisboa: ICS - Imprensa de Ciências Sociais, 2011.

Saha, Somnath, Sanjay Saint, and Dimitri A. Christakis. "Impact Factor: A Valid Measure of Journal Quality?". Journal of the Medical Library Association 91, no. $1(05 / /$ received

08//accepted 2003): 42-46.

Santos, Lurdes C., Cândida F. Abreu, Sandra M. Xerinda, Margarida Tavares, Raquel Lucas, and António C. Sarmento. "Severe Imported Malaria in an Intensive Care Unit: A Review of 59 Cases." Malaria Journal 11, no. 1 (2012): 1-9.

Santos Pereira, Tiago. "International Dimension of Research in Portugal: Implications for Coordination and Collaboration in European Research." Science and Public Policy 29, no. 6 (2002): 451-61.

. "Uma Análise Do Impacto Das Políticas Europeias Na Colaboração Internacional Em Investigação Científica Em Portugal E No Reino Unido." Análise Social XXXI (1. ${ }^{\circ}$ ), no. 135 (1996): 229-65.

Saraiva, Tiago, Ana Delicado, and Cristiana Bastos. "Configurações Da Investigação Científica Em Portugal: Três Estudos De Caso." In Itinerários. A Investigação Nos 25 Anos Do Ics, edited by Filipe Carreira da Silva, Karin Wall, Manuel 
Villaverde Cabral and Sofia Aboim, 429-50. Lisboa: Imprensa de Ciências Sociais, 2008.

Slaughter, Sheila, and Larry L. Leslie. Academic Capitalism: Politics, Policies, and the Entrepreneurial University. Johns Hopkins University Press, 1999.

Trouiller, Patrice, Piero Olliaro, Els Torreele, James Orbinski, Richard Laing, and Nathan Ford. "Drug Development for Neglected Diseases: A Deficient Market and a Public-Health Policy Failure." The Lancet 359, no. 9324 (6/22/ 2002): 2188-94.

WHO. World Malaria Report 2014. Geneva: World Health Organization Library Cataloguing-in-Publication Data, 2014.

Ziman, John. Real Science: What It Is and What It Means. Cambridge University Press, 2002.

Data de receção: 29 de Fevereiro de 2016; Data de aceitação: 3 de Abril de 2016 